\title{
On sound ranging in proper metric spaces
}

\author{
Sergij V. Goncharov
}

\begin{abstract}
We consider the sound ranging, or source localization, problem - find the source-point from the moments when the wave-sphere of linearly, with time, increasing radius reaches the sensor-points - in proper metric spaces (any closed ball is compact) and, in particular, in the finite-dimensional normed spaces. We approximate the solution to arbitrary precision by the iterative process with the stopping criterion. Implementation of the proposed method in Julia language is included.
\end{abstract}

\section{Introduction}

Let $(X ; \rho)$ be a metric space with the metric $\rho: X \times X \rightarrow \mathbb{R}_{+}$. Let $\mathbf{s} \in X$ be an unknown "source". At unknown moment $t_{0} \in \mathbb{R}$ of time the source "emits the (sound) wave", which is the sphere $\left\{\mathbf{x} \in X \mid \rho(\mathbf{x} ; \mathbf{s})=v\left(t-t_{0}\right)\right\}$ for any moment $t \geqslant t_{0}$. We assume that "sound speed" $v=1$.

Let $\left\{\mathbf{r}_{i}\right\}_{i \in I}, \mathbf{r}_{i} \in X$, be an indexed set of known "sensors". For each sensor we know the moment $t_{i}$ when it was reached by the expanding wave; that is, $t_{i}=t_{0}+\rho\left(\mathbf{r}_{i} ; \mathbf{s}\right)$ are known. The sound ranging problem (SRP), also called source localization, is to find $\mathbf{s}$ and $t_{0}$ from known $\left(\left\{\mathbf{r}_{i}\right\} ;\left\{t_{i}\right\}\right)$.

SRPs of this and more general forms, usually in Euclidean space, appear, inter alia, in acoustics [38], geophysics [34], navigation [4], (wireless) sensor networks [25]; there is an abundant literature on the subject and on the proposed techniques, see, e.g., [9, Section 1], [2, Section 9.1] for further references.

In [15] we investigated noiseless SRP in the infinite-dimensional separable Hilbert space $H$. The method there was the "classical" one applied in $\mathbb{R}_{2}^{m}$, solve the set of implied equations $\left(t_{i}-t_{0}\right)^{2}=\rho^{2}\left(\mathbf{r}_{i} ; \mathbf{s}\right)=\sum_{j}\left(r_{j}^{(i)}-s_{j}\right)^{2}$, where

Received April 10, 2019.

2010 Mathematics Subject Classification. 41A65, 54E50, 46B20, 40A05, 68W25.

Key words and phrases. Source localization, approximation, algorithm, proper metric space, normed space, finite-dimensional, implementation.

https://doi.org/10.12697/ACUTM.2020.24.14 
$t_{0}$ and coordinates $\left\{s_{j}\right\}_{j \in \mathbb{N}}$ of $\mathbf{s}$ are unknowns, - with few technicalities related to countability of the coordinates. It is a "solving" method: we express exact values of $s_{j}$ through known $t_{i}$ and $r_{j}^{(i)}$, in closed form.

This time we look into another generalization of SRP, without Euclidicity in general. The classical approach does not work anymore, because even if there are coordinates, they are not so easily "extractable" from the equations $t_{i}-t_{0}=\rho\left(\mathbf{r}_{i} ; \mathbf{s}\right)$, which become significantly nonlinear.

Instead, we describe the iterative process that "converges" to the source in certain sense explained further; this is an "approximating" method. In short, we cover the regions of the space by balls, and repeatedly refine the cover by a) replacing every ball with its cover by the balls of halved radius, then b) removing from the cover each ball such that certain "defect" at its center is greater than its doubled radius. "Defect" at the source is 0 .

It is presented as an algorithm. How practical such algorithm is depends on its "executor": what and how many elementary computations the executor is allowed to perform. If e.g. we had a "computer" $\mathfrak{U}$ with card $X$ cores, we would plainly assign to each core single $\mathbf{x} \in X$ to verify if $\mathbf{x}$ is a solution $\left(t_{i}-\rho\left(\mathbf{r}_{i} ; \mathbf{x}\right) \equiv\right.$ const, see below $)$. But $\mathfrak{U}$ is too "heavy".

Our target executor is closer to the nowadays "general purpose" computing devices, whose natural limitations on memory and time make $\mathfrak{U}$-approach infeasible. We concretize the computation model in Subsection 1.2. In Appendix we provide the proof-of-concept copy-paste-execute implementation of the algorithm in Julia for $X=\mathbb{R}_{p}^{m}$.

Some examples show the "real world" problems to which the described method can, in principle, be applied.

Disclaimer. The intent of this paper is not to proclaim the (dubious) "novelty" of the method being proposed, but to develop the approach of akin methods in more general context and watch how it works. On the other hand, we have not found an algorithm to solve SRP in general metric spaces in the literature available to us.

Focusing rather on rigour, we consider "empty" spaces without "physics" - echoes, varying sound speed, noise (see Remark 3).

Root finding versus optimization. Searching for $\operatorname{argmin} f(\mathbf{x})$ (or for $\operatorname{argmax}(-f(\mathbf{x})))$ is the optimization problem that is part of the most of approaches to solve SRPs, especially with noised measurements $(f(\mathbf{x})$ is "cost" / "plausibility" function there). It is performed either (1-stage) directly in the space of possible source positions to estimate the actual position, or (2-stage) in the space of relative time-delays $t_{i}-t_{j}$ between sensors to estimate these delays, which then allow to obtain the source position in closed form or, alternatively, estimate it as well (see [1], [5], [7], [14], [2]). For example, in [1] the branch \& bound technique is applied and compared 
to other ones. The maximum likelihood estimator is, too, one of common approaches to such optimization, though there are issues with local minima when the cost function is not strictly concave (see [2, Section 9.4]). The Euclidicity of the space where the wave propagates is important in deriving the closed form solutions and in the least squares localizations (see [5, Section 4] and [2, Section 9.5]).

In our simplified case the exact relative delays are known and a nonnegative function has a unique zero; we search for that zero, rather than extremum, in the (non-Euclidean) space of possible source positions. This is a root finding of "bracketing", or "exclude \& enclose", type (see [6, Section $1.2])$.

The bibliography with somewhat more emphasis on the practice of sound ranging, including historical surveys, was given in [15], or better, see [37], [9], [2, Chapter 9], [23, pp. 320-323].

The symbol indicates the assumptions, or constraints, that we require to hold unless stated otherwise. But the symbol $\bullet$ is for the statements that are considered to be well-known under given assumptions and included for the sake of completeness, without proofs or references.

\section{SRP in proper metric spaces}

\subsection{Preliminaries.}

- "2nd $\triangle$-inequality": for any $\mathbf{x}, \mathbf{y}, \mathbf{z} \in X|\rho(\mathbf{x} ; \mathbf{z})-\rho(\mathbf{z} ; \mathbf{y})| \leqslant \rho(\mathbf{x} ; \mathbf{y})$.

As usual, $\mathbf{x}_{k} \underset{k \rightarrow \infty}{\longrightarrow} \mathbf{y}$ means the convergence $\rho\left(\mathbf{x}_{k} ; \mathbf{y}\right) \underset{k \rightarrow \infty}{\longrightarrow} 0$.

- Continuity of metric: $\mathbf{x}_{k} \underset{k \rightarrow \infty}{\longrightarrow} \mathbf{y}$ implies $\rho\left(\mathbf{x}_{k} ; \mathbf{z}\right) \underset{k \rightarrow \infty}{\longrightarrow} \rho(\mathbf{y} ; \mathbf{z})$.

$B(\mathbf{c} ; r)=\{\mathbf{x} \in X \mid \rho(\mathbf{x} ; \mathbf{c})<r\}$ and $B[\mathbf{c} ; r]=\{\mathbf{x} \in X \mid \rho(\mathbf{x} ; \mathbf{c}) \leqslant r\}$ denote the open and closed balls with center $\mathbf{c}$ and of radius $r$.

- For any $B[\mathbf{c} ; r]$ and any $\mathbf{a} \in X$ such that $\rho(\mathbf{a} ; \mathbf{c})=d$, we have $d-r \leqslant$ $\rho(\mathbf{x} ; \mathbf{a}) \leqslant d+r$ for any $\mathbf{x} \in B[\mathbf{c} ; r]$.

The set $A \subseteq X$ is said to be compact if for any sequence $\left\{\mathbf{x}_{k}\right\}_{k \in \mathbb{N}} \subseteq A$ there exists a subsequence $\left\{\mathbf{x}_{k_{l}}\right\}_{l \in \mathbb{N}}$ such that $\mathbf{x}_{k_{l}} \underset{l \rightarrow \infty}{\longrightarrow} \mathbf{x}_{0} \in A$.

- If $A$ is compact, then any closed subset of $A$ is compact too.

The family of sets $\left\{C_{j}\right\}_{j \in J}, C_{j} \subseteq X$, is said to be a cover of $A \subseteq X$ if $A \subseteq \bigcup_{j \in J} C_{j}$

- The closed $A \subseteq X$ is compact if and only if any open cover of $A$ has a finite subcover.

The set $A \subseteq X$ is called bounded if $\operatorname{diam} A=\sup _{\mathbf{x}, \mathbf{y} \in A} \rho(\mathbf{x} ; \mathbf{y})<\infty$.

कM1. $(X ; \rho)$ is proper: any closed ball is compact. 
Such spaces are also called finitely compact or having the Heine-Borel property ([10, Section 1.5, p. 43], [31, Section 1.4, p. 32]; in addition, see also [40]).

- In M1, "any closed ball" can be replaced with "any closed and bounded subset".

- A proper metric space is complete: any fundamental sequence converges. In fact, it would suffice that $\delta>0$ exists such that, for any $\mathbf{x} \in X, B[\mathbf{x} ; \delta]$ is compact. We remark that the converse fails: the infinite-dimensional separable Hilbert space is complete, but not proper. Moreover, any infinitedimensional normed space is not proper (F. Riesz, [22, Section 6.24]).

Now we proceed to the SRP.

The source $\mathbf{s} \in X$ and the emission moment $t_{0} \in \mathbb{R}$ are unknown.

201. The set of sensors is finite: $\left\{\mathbf{r}_{i}\right\}_{i=1}^{n}, \mathbf{r}_{i} \in X$, and $\mathbf{r}_{i} \neq \mathbf{r}_{j}$ if $i \neq j$.

These sensors and the moments

$$
t_{i}=t_{0}+\rho\left(\mathbf{r}_{i} ; \mathbf{s}\right), \quad i=\overline{1, n}
$$

define the SRP $\left(\left\{\mathbf{r}_{i}\right\} ;\left\{t_{i}\right\}\right)$. Each pair $\left(\mathbf{s}^{\prime} ; t^{\prime}\right)$ satisfying the set of equations

$$
t_{i}=t^{\prime}+\rho\left(\mathbf{r}_{i} ; \mathbf{s}^{\prime}\right), \quad i=\overline{1, n}
$$

is a solution of this SRP. Since $t^{\prime}$ is defined uniquely from any such equation for given $\mathbf{s}^{\prime}$, the source $\mathbf{s}^{\prime}$ itself can be called a solution too.

2. 2 . The solution $\mathbf{s}$ of the $\operatorname{SRP}\left(\left\{\mathbf{r}_{i}\right\} ;\left\{t_{i}\right\}\right)$ is unique.

Obviously, $\mathbf{2} 2$ is not true in general. For $n=1$ any $\mathbf{y} \in X$ is a solution, with $t^{\prime}=t_{1}-\rho\left(\mathbf{y} ; \mathbf{r}_{1}\right)$. In $\mathbb{R}_{2}^{2}$ we can place "true" and "false" sources, $\mathbf{s}$ and $\mathbf{s}^{\prime}$ respectively, at the two foci of a hyperbola, and place 3 sensors on the same branch of that hyperbola. Then $\rho\left(\mathbf{r}_{i} ; \mathbf{s}\right)-\rho\left(\mathbf{r}_{i} ; \mathbf{s}^{\prime}\right) \equiv d$, thus $\mathbf{s}^{\prime}$ emitting the wave at the moment $t^{\prime}=t_{0}+d$ is another solution.

The solution in $\mathbb{R}_{2}^{m}$ is unique if we take $m+2$ sensors such that $\left\{\mathbf{r}_{2}-\mathbf{r}_{1}\right.$; $\left.\ldots ; \mathbf{r}_{m+1}-\mathbf{r}_{1}\right\}$ is a basis of $\mathbb{R}^{m}$ and $\mathbf{r}_{m+2}=2 \mathbf{r}_{1}-\mathbf{r}_{2}$ (see [15, Proposition 4]).

Definition 1.1. For any $\mathrm{x} \in X$ the backward moments are

$$
\tau_{i}(\mathbf{x}):=t_{i}-\rho\left(\mathbf{x} ; \mathbf{r}_{i}\right), \quad i=\overline{1, n} .
$$

$\tau_{i}(\mathbf{x})$ is the moment when the wave must be emitted from $\mathbf{x}$ to reach $\mathbf{r}_{i}$ at the moment $t_{i}$.

Definition 1.2. For any $\mathbf{x} \in X$ the defect is

$$
D(\mathbf{x}):=\frac{1}{n} \sum_{i=1}^{n}\left|\tau_{i}(\mathbf{x})-\frac{1}{n} \sum_{j=1}^{n} \tau_{j}(\mathbf{x})\right| .
$$

(Compare with [5, Section 3] or [33, Section 2.4].) We rewrite

$$
D(\mathbf{x})=\frac{1}{n^{2}} \sum_{i=1}^{n}\left|\sum_{j=1}^{n}\left[\tau_{i}(\mathbf{x})-\tau_{j}(\mathbf{x})\right]\right| \leqslant \frac{1}{n^{2}} \sum_{i=1}^{n} \sum_{j=1}^{n}\left|\tau_{i}(\mathbf{x})-\tau_{j}(\mathbf{x})\right| .
$$

The elementary properties of $D(\cdot)$ follow (Propositions 1.1-1.4). 
Proposition 1.1. An element $\mathbf{s}^{\prime} \in X$ is a solution of the $S R P$ if and only if $D\left(\mathbf{s}^{\prime}\right)=0$.

Proof. If $\mathbf{s}^{\prime}$ is a solution, then $t_{i}=t^{\prime}+\rho\left(\mathbf{r}_{i} ; \mathbf{s}^{\prime}\right), \tau_{i}\left(\mathbf{s}^{\prime}\right) \equiv t^{\prime}$. So $\tau_{i}\left(\mathbf{s}^{\prime}\right)-\tau_{j}\left(\mathbf{s}^{\prime}\right) \equiv$ 0 , thus $D\left(\mathbf{s}^{\prime}\right)=0$. Contrariwise, $D\left(\mathbf{s}^{\prime}\right)=0$ implies $\tau_{i}\left(\mathbf{s}^{\prime}\right) \equiv t^{\prime}=\frac{1}{n} \sum_{j=1}^{n} \tau_{j}\left(\mathbf{s}^{\prime}\right)$, and $\left(\mathbf{s}^{\prime} ; t^{\prime}\right)$ is a solution.

Corollary 1.1. $D(\mathbf{x})$ has exactly one zero in $X$, at $\mathbf{x}=\mathbf{s}^{\prime}$.

Proposition 1.2. For any $\mathbf{x} \in X,\left|\tau_{i}(\mathbf{x})-\tau_{j}(\mathbf{x})\right| \geqslant D(\mathbf{x})$ for some $i, j$.

Proof. Assuming the contrary, we have $D(\mathbf{x})<\frac{1}{n^{2}} \sum_{i=1}^{n} \sum_{j=1}^{n} D(\mathbf{x})=D(\mathbf{x})$ which is a contradiction.

Proposition 1.3. For any $\mathbf{x}, \mathbf{y} \in X,|D(\mathbf{x})-D(\mathbf{y})| \leqslant 2 \rho(\mathbf{x} ; \mathbf{y})$.

Proof. We have

$$
\begin{aligned}
|D(\mathbf{x})-D(\mathbf{y})| & \leqslant \frac{1}{n^{2}} \sum_{i=1}^{n}\left|\sum_{j=1}^{n}\left[\tau_{i}(\mathbf{x})-\tau_{j}(\mathbf{x})-\left\{\tau_{i}(\mathbf{y})-\tau_{j}(\mathbf{y})\right\}\right]\right| \\
& \leqslant \frac{1}{n^{2}} \sum_{i=1}^{n} \sum_{j=1}^{n}\left|\left[\tau_{i}(\mathbf{x})-\tau_{i}(\mathbf{y})\right]-\left[\tau_{j}(\mathbf{x})-\tau_{j}(\mathbf{y})\right]\right| \\
& \leqslant \frac{1}{n^{2}} \sum_{i, j}\left[\left|\tau_{i}(\mathbf{x})-\tau_{i}(\mathbf{y})\right|+\left|\tau_{j}(\mathbf{x})-\tau_{j}(\mathbf{y})\right|\right] \\
& =\frac{1}{n^{2}} \sum_{i, j}\left[\left|\rho\left(\mathbf{x} ; \mathbf{r}_{i}\right)-\rho\left(\mathbf{y} ; \mathbf{r}_{i}\right)\right|+\left|\rho\left(\mathbf{x} ; \mathbf{r}_{j}\right)-\rho\left(\mathbf{y} ; \mathbf{r}_{j}\right)\right|\right] \\
& \leqslant \frac{1}{n^{2}} \sum_{i, j}[\rho(\mathbf{x} ; \mathbf{y})+\rho(\mathbf{x} ; \mathbf{y})]=2 \rho(\mathbf{x} ; \mathbf{y}) .
\end{aligned}
$$

It should be noted that $D(\cdot)$ is a Lipschitz function (see [17, Chapter 6], [35, Section 9.4]).

Corollary 1.2. $D(\mathbf{x})$ is uniformly continuous on $X$.

Proposition 1.4. Let $\mathbf{s} \in B=B[\mathbf{c} ; r]$. For any $\delta>0$, there exists $\varepsilon=\varepsilon(\delta)>0$ such that if $\mathbf{x} \in B$ and $D(\mathbf{x})<\varepsilon$, then $\rho(\mathbf{x} ; \mathbf{s})<\delta$.

Proof. Let $S=\{\mathbf{x} \in B \mid \rho(\mathbf{x} ; \mathbf{s}) \geqslant \delta\}$ and $\varepsilon=\inf _{\mathbf{x} \in S} D(\mathbf{x})$. We claim that $S$ is compact. Indeed, $S \subset B$ and $S$ is closed due to continuity of metric. Since for all $\mathbf{x} \in S, D(\mathbf{x})>0$ due to Corollary 1.1, we have $\varepsilon>0$ (otherwise for any $k \in \mathbb{N}$ there exists $\mathbf{x}_{k} \in S$ with $D\left(\mathbf{x}_{k}\right)<\frac{1}{k}$, and it follows from compactness 
of $S$ that there exists $\left\{\mathbf{x}_{k_{l}}\right\}_{l \in \mathbb{N}}$ such that $\mathbf{x}_{k_{l}} \longrightarrow \mathbf{x}_{0} \in S$. Then, since $D(\mathbf{x})$ is continuous, so $D\left(\mathbf{x}_{k_{l}}\right) \underset{l \rightarrow \infty}{\longrightarrow} D\left(\mathbf{x}_{0}\right)$, but $0 \leqslant D\left(\mathbf{x}_{k_{l}}\right)<\frac{1}{k_{l}} \leqslant \frac{1}{l}$ implies $D\left(\mathbf{x}_{0}\right)=\lim _{l \rightarrow \infty} D\left(\mathbf{x}_{k_{l}}\right)=0-$ a contradiction $)$.

Now, if $\mathbf{x} \in B$ and $D(\mathbf{x})<\varepsilon$, then $\mathbf{x} \notin S$, which means $\rho(\mathbf{x} ; \mathbf{s})<\delta$.

Test for a ball. Consider arbitrary $B=B[\mathbf{c} ; r] \subseteq X$. If $\mathbf{s} \in B$, then $\rho\left(\mathbf{r}_{i} ; \mathbf{c}\right)-r \leqslant \rho\left(\mathbf{r}_{i} ; \mathbf{s}\right) \leqslant \rho\left(\mathbf{r}_{i} ; \mathbf{c}\right)+r, i=\overline{1, n}$, which is equivalent to $t_{0}=t_{i}-\rho\left(\mathbf{r}_{i} ; \mathbf{s}\right) \in\left[t_{i}-\rho\left(\mathbf{r}_{i} ; \mathbf{c}\right)-r ; t_{i}-\rho\left(\mathbf{r}_{i} ; \mathbf{c}\right)+r\right], i=\overline{1, n}$.

Hence $t_{0} \in C=\bigcap_{i=1}^{n}\left[\tau_{i}(\mathbf{c})-r ; \tau_{i}(\mathbf{c})+r\right] \neq \varnothing$.

It is easy to see that $C \neq \varnothing$ if and only if the following inequality holds:

$$
2 r \geqslant \max _{i} \tau_{i}(\mathbf{c})-\min _{i} \tau_{i}(\mathbf{c})=\max _{i, j}\left|\tau_{i}(\mathbf{c})-\tau_{j}(\mathbf{c})\right|=: I(\mathbf{c}) .
$$

Thus we have the inference: if $\mathbf{s} \in B[\mathbf{c} ; r]$, then $2 r \geqslant I(\mathbf{c})$. Conversely, $2 r<I(\mathbf{c})$ implies $\mathbf{s} \notin B[\mathbf{c} ; r]$.

Since $\left|\tau_{i}(\mathbf{c})-\tau_{j}(\mathbf{c})\right| \leqslant I(\mathbf{c})$, we have $D(\mathbf{c}) \leqslant \frac{1}{n^{2}} \sum_{1 \leqslant i, j \leqslant n} I(\mathbf{c})=I(\mathbf{c})$. So if $2 r<D(\mathbf{c})$, then $\mathbf{s} \notin B[\mathbf{c} ; r]$

and the condition " $2 r<D(\mathbf{c})$ " divides the family of all closed balls in $X$ into 2 families:

1) $\mathcal{N}$ (egative) - the balls that satisfy it and thus do not contain $\mathbf{s}$,

2) $\mathcal{S}$ (uspicious) - the balls that do not satisfy it.

Of course, even if $B \in \mathcal{S}$, more "advanced" tests may prove that $\mathbf{s} \notin B$.

Proposition 1.5. If $D(\mathbf{x})>0, \mathbf{x} \in B=B[\mathbf{y} ; r]$, and $r<\frac{1}{4} D(\mathbf{x})$, then $B \in \mathcal{N}$.

Proof. By Proposition 1.3, it follows from $\rho(\mathbf{y} ; \mathbf{x}) \leqslant r<\frac{1}{4} D(\mathbf{x})$ that $\mid D(\mathbf{y})-$ $D(\mathbf{x}) \mid<\frac{1}{2} D(\mathbf{x})$. Thus $D(\mathbf{y})>\frac{1}{2} D(\mathbf{x})>0$. Since $r<\frac{1}{4} D(\mathbf{x})<\frac{1}{2} D(\mathbf{y}),(3)$ implies $B \in \mathcal{N}$.

If $D(\mathbf{x})>0$, then small enough suspicious balls do not contain $\mathbf{x}$.

Refining cover. Consider any $B=B[\mathbf{z} ; r] . \mathcal{A}_{0}=\left\{B\left(\mathbf{c} ; \frac{r}{2}\right) \mid \mathbf{c} \in B\right\}$ is a cover of $B$ by open sets. From $\$$ M1 it follows that there exists a finite subcover $\mathcal{A}_{1}=\left\{B\left(\mathbf{c}_{i} ; \frac{r}{2}\right) \mid i=\overline{1, N}\right\} \subseteq \mathcal{A}_{0}$, which also covers $B$. Clearly, $\mathcal{B}=\left\{B\left[\mathbf{c}_{i} ; \frac{r}{2}\right] \mid i=\overline{1, N}\right\}$ is a finite cover of $B$ too. Thus we have obtained

Proposition 1.6. For any $B=B[\mathbf{z} ; r]$ there exists a finite cover $\mathcal{B}=$ $\mathcal{B}(B)=\left\{B\left[\mathbf{c}_{i} ; \frac{r}{2}\right] \mid i=\overline{1, N}\right\}$ of $B$, and for any $B\left[\mathbf{c} ; \frac{r}{2}\right] \in \mathcal{B}, \rho(\mathbf{c} ; \mathbf{z}) \leqslant r$.

Naturally, we want $N$ to be as small as possible; however, the time spent in the (intricate) positioning of less balls can exceed the time gained by not testing more balls. We omit this topic here; see [11], [16, Chapter 2]. If $N_{d} \in \mathbb{N}$ exists such that any $B[\mathbf{z} ; r]$ can be covered by at most $N_{d}$ closed balls 
of radius $\frac{r}{2}$, then $(X ; \rho)$ is called doubling, and $N_{d}$ is its doubling constant (see $[17$, p. 81]).

For the method at hand we can weaken $\rho(\mathbf{c} ; \mathbf{z}) \leqslant r$ to

$$
\rho(\mathbf{c} ; \mathbf{z}) \leqslant K_{1} r, K_{1} \geqslant 1 .
$$

When, for example, points of $X$ are provided with coordinates, we can make $\mathcal{B}$ in a more "constructive" way. In particular, next section describes this procedure in the finite-dimensional normed spaces. Other examples are Riemannian manifolds with intrinsic metric (see [10, Sections 7.1, 8.1], [32, Section 3.3]), optionally immersed in $\mathbb{R}_{2}^{m}$, and some graphs whose edges have lengths.

Let $\mathcal{B}_{0}=\{B[\mathbf{z} ; r]\}, \mathcal{B}_{1}=\mathcal{B}(B[\mathbf{z} ; r])$. Then let $\mathcal{B}_{2}$ be the union of covers of all balls from $\mathcal{B}_{1}, \ldots, \mathcal{B}_{k}$ be the union of covers of all balls from $\mathcal{B}_{k-1}, \ldots$

It is evident that the balls in $\mathcal{B}_{k}$ are of radius $\frac{r}{2^{k}}$ and $\bigcup_{B \in \mathcal{B}_{k}} B \subseteq \bigcup_{B \in \mathcal{B}_{k+1}} B$.

Proposition 1.7. Let $\mathcal{B}_{\infty}=\bigcup_{k=0}^{\infty} \mathcal{B}_{k}$. Then

where $K=2 K_{1}+1$.

$$
\bigcup_{B \in \mathcal{B}_{\infty}} \stackrel{k=0}{B} \subseteq B[\mathbf{z} ; K r]
$$

Proof. For any $B^{\prime}=B\left[\mathbf{z}_{k} ; \frac{r}{2^{k}}\right] \in \mathcal{B}_{k}$ there is $B^{\prime \prime}=B\left[\mathbf{z}_{k-1} ; \frac{r}{2^{k-1}}\right] \in \mathcal{B}_{k-1}$ such that $B^{\prime} \in \mathcal{B}\left(B^{\prime \prime}\right)$ (by construction). So by (4), $\rho\left(\mathbf{z}_{k} ; \mathbf{z}_{k-1}\right) \leqslant K_{1} \cdot \frac{r}{2^{k-1}}=$ $2 K_{1} r \cdot \frac{1}{2^{k}}$. Therefore,

$$
\begin{aligned}
\rho\left(\mathbf{z}_{k} ; \mathbf{z}\right) & \leqslant \rho\left(\mathbf{z}_{k} ; \mathbf{z}_{k-1}\right)+\rho\left(\mathbf{z}_{k-1} ; \mathbf{z}\right) \leqslant 2 K_{1} r \cdot \frac{1}{2^{k}}+\rho\left(\mathbf{z}_{k-1} ; \mathbf{z}\right) \\
& \leqslant 2 K_{1} r\left(\frac{1}{2^{k}}+\frac{1}{2^{k-1}}\right)+\rho\left(\mathbf{z}_{k-2} ; \mathbf{z}\right) \leqslant \ldots \\
& \leqslant 2 K_{1} r \sum_{i=1}^{k} 2^{-i}+\rho(\mathbf{z} ; \mathbf{z}) \leqslant 2 K_{1} r .
\end{aligned}
$$

Now, for any $\mathbf{x} \in \bigcup_{B \in \mathcal{B}_{\infty}} B: B\left[\mathbf{z}_{k} ; \frac{r}{2^{k}}\right]$ exists that contains $\mathbf{x}$ for some $k$, hence $\rho(\mathbf{x} ; \mathbf{z}) \leqslant \rho\left(\mathbf{x} ; \mathbf{z}_{k}\right)+\rho\left(\mathbf{z}_{k} ; \mathbf{z}\right) \leqslant \frac{r}{2^{k}}+2 K_{1} r \leqslant\left(2 K_{1}+1\right) r$.

\subsection{Method.}

First, we construct the sequence of points that converges to s, using $\left(\left\{\mathbf{r}_{i}\right\} ;\left\{t_{i}\right\}\right)$. Then we add the criterion to halt this process when required precision is attained. Finally, we describe the "real world" computer able to compute the sequence up to the halt.

We add one more assumption for the sake of simplicity:

23. At least one ball $B_{0,1}=B\left[\mathbf{c}_{0,1} ; r\right] \ni \mathbf{s}$ is known.

( $B_{0,1}$ is a "big enough" ball that contains all possible source positions.) 
Step 0. Let $k=0, \mathcal{C}_{0}=\left\{B_{0,1}\right\}$, and $r_{0}=r$.

Step 1. Let $\mathcal{C}_{k+1}=\varnothing$.

For each ball $B=B\left[\mathbf{y} ; r_{k}\right] \in \mathcal{C}_{k}, r_{k}=\frac{r}{2^{k}}$, there is the cover $\mathcal{B}$ of $B$, which consists of the balls $B^{\prime}=B\left[\mathbf{c} ; r_{k+1}\right], r_{k+1}=\frac{1}{2} r_{k}=\frac{r}{2^{k+1}}$.

Consider each $B^{\prime}$ and apply test (3) to it. If $B^{\prime} \in \mathcal{S}$, then add $B^{\prime}$ to $\mathcal{C}_{k+1}$.

Step 2. Let $\mathbf{z}_{k+1}$ be the center of the arbitrarily chosen ball from $\mathcal{C}_{k+1}$.

Step 3. $k:=k+1$, goto Step 1 .

It follows from $\mathbf{s} \in B_{0,1} \subseteq \bigcup_{B \in \mathcal{B}\left(B_{0,1}\right)} B$ that at least one ball from $\mathcal{B}\left(B_{0,1}\right)$ contains $\mathbf{s}$, this ball belongs to $\mathcal{S}$ and therefore $\mathcal{C}_{1} \neq \varnothing$. Similarly, at least one ball from $\bigcup_{B \in \mathcal{C}_{1}} \mathcal{B}(B)$ contains $\mathbf{s}$, implying $\mathcal{C}_{2} \neq \varnothing$, and so on: for any $k \in \mathbb{Z}_{+} \mathcal{C}_{k} \neq \varnothing$. Hence these steps define the infinite sequence of the covers $\left\{\mathcal{C}_{k}\right\}_{k=0}^{\infty}$ and the infinite sequence of the ball centers $\left\{\mathbf{z}_{k}\right\}_{k=1}^{\infty}$.

Proposition 1.8. One has $\mathbf{z}_{k} \underset{k \rightarrow \infty}{\longrightarrow} \mathbf{s}$.

Proof. By Proposition 1.7, $\left\{\mathbf{z}_{k}\right\}_{k \in \mathbb{N}} \subseteq \widehat{B}=B\left[\mathbf{c}_{0,1} ; K r\right]$ and $\mathbf{s} \in B_{0,1} \subseteq \widehat{B}$.

Take any $\delta>0$. By Proposition 1.4, there exists $\varepsilon>0$ such that if $\mathbf{x} \in \widehat{B}$ and $D(\mathbf{x})<\varepsilon$, then $\rho(\mathbf{x} ; \mathbf{s})<\delta$. Since $r_{k}=\frac{r}{2^{k}} \underset{k \rightarrow \infty}{\longrightarrow} 0$, for any $k \geqslant k_{0}=2+\left\lfloor\log _{2} \frac{r}{\varepsilon}\right\rfloor, r_{k}<\frac{1}{2} \varepsilon$. By construction, for any $B=B\left[\mathbf{c} ; r_{k}\right] \in \mathcal{C}_{k}$ : $B \in \mathcal{S}$, so $D(\mathbf{c}) \leqslant 2 r_{k}<\varepsilon$. Hence $\rho(\mathbf{c} ; \mathbf{s})<\delta$; in particular, $\rho\left(\mathbf{z}_{k} ; \mathbf{s}\right)<\delta$.

In practice, however, we would like to know when to halt this process. We need a discernible "sign" that $\mathbf{z}_{k}$ is close enough to $\mathbf{s}, \rho\left(\mathbf{z}_{k} ; \mathbf{s}\right)<\delta$ for the preselected precision $\delta$. And we do not rely on the condition $r_{k}<\frac{1}{2} \varepsilon$, because $\varepsilon$ is, in a sense, unknown - defined "not constructively enough"; put differently, the convergence rate is unknown.

Therefore, we add the stopping criterion to Step 3, replacing it by

Step 3'. $k:=k+1$. Let $d_{k}=r_{k}+\max _{B\left[\mathbf{c} ; r_{k}\right] \in \mathcal{C}_{k}} \rho\left(\mathbf{z}_{k} ; \mathbf{c}\right)$. If $d_{k}<\delta$, then halt; else goto Step 1.

By Proposition 1.4, there exists $\varepsilon>0$ : if $\mathbf{x} \in \widehat{B}$ and $D(\mathbf{x})<\varepsilon$, then $\rho(\mathbf{x} ; \mathbf{s})<\frac{1}{4} \delta$. When $r_{k}=\frac{r}{2^{k}}<\frac{1}{2} \varepsilon$, for two balls $B\left[\mathbf{z}_{k} ; r_{k}\right], B\left[\mathbf{c} ; r_{k}\right]$ to be in $\mathcal{C}_{k} \subseteq \mathcal{S}$ it is necessary that $D\left(\mathbf{z}_{k}\right), D(\mathbf{c}) \leqslant 2 r_{k}<\varepsilon$, thus $\rho\left(\mathbf{z}_{k} ; \mathbf{c}\right) \leqslant \rho\left(\mathbf{z}_{k} ; \mathbf{s}\right)+$ $\rho(\mathbf{s} ; \mathbf{c})<\frac{1}{2} \delta$, - for large enough $k$ the condition $\max _{B\left[\mathbf{c} ; r_{k}\right] \in \mathcal{C}_{k}} \rho\left(\mathbf{z}_{k} ; \mathbf{c}\right)<\frac{1}{2} \delta$ holds.

Obviously, the condition $r_{k}<\frac{1}{2} \delta$ holds when $k>\log _{2} \frac{2 r}{\delta}$.

As soon as the process reaches $k$ such that both conditions hold, $d_{k}<\delta$ and it halts. Whenever it halts (maybe even for smaller $k$ ), $\mathbf{z}_{k}$ is the sought approximation of $\mathbf{s}$ : suppose $\mathbf{s} \in B\left[\mathbf{c} ; r_{k}\right] \in \mathcal{C}_{k}$, then

$$
\rho\left(\mathbf{z}_{k} ; \mathbf{s}\right) \leqslant \rho\left(\mathbf{z}_{k} ; \mathbf{c}\right)+\rho(\mathbf{c} ; \mathbf{s}) \leqslant d_{k}<\delta .
$$


We call the 0-1-2-3' algorithm "refining cover by defect", RCD.

We would be glad to know (and thank everyone who will point out) who and when has proposed it already, especially in general metric spaces. Perhaps it happened as early as 1920-40s?

Computation model. Consider the computer $\mathfrak{C}$ that:

a) performs 4 operations of arithmetic on $\mathbb{R}$ with high enough precision;

b) is able to store in its memory a reasonably large number $N \in \mathbb{N}$ of $X$ points (perhaps in coordinate form);

c) calculates $\rho\left(\mathbf{x}^{\prime} ; \mathbf{x}^{\prime \prime}\right)$ for any given $\mathbf{x}^{\prime}, \mathbf{x}^{\prime \prime} \in X$ and builds the finite cover $\mathcal{B}$ of any given ball $B$ in a time not greater than $T \in \mathbb{R}_{+}$.

(Nothing beyond Turing machine with finite tape and its equivalents, see [18, Section 8.6.2].)

We claim that $\mathfrak{C}$ executes $\mathrm{RCD}$ in a finite time; indeed, the steps $0,1,2,3$ ' involve only a finite number of cover building, distance calculation, and real arithmetic. The exact amount of this time and the actual error $\rho\left(\mathbf{z}_{k} ; \mathbf{s}\right)<\delta$ depend on properties (for example, dimensionality) of $X$, arrangement of sensors, and so on.

We do not claim that RCD is optimal for $\mathfrak{C}$ ("classical" approach, for one, is faster in $\mathbb{R}_{2}^{m}$ ); however, as soon as $X$ is infinite $\left(\operatorname{card} X \geqslant \aleph_{0}\right)$ and unbounded, "test every point in $X$ " becomes infeasible on $\mathfrak{C}$, while RCD can be implemented. In other words, RCD lessens the complexity of computation from infinite to finite (and makes it more "directed"). The readers concerned with actual practicality are invited to run the code from Appendix.

Speaking of optimality, in certain proper metric spaces, with certain arrangement of sensors, provably more efficient methods may exist. We also remark that Step 1 is straightforwardly parallelizable.

\section{SRP in finite-dimensional normed spaces}

Now we denote by $(X ;\|\cdot\|)$ a normed space over the field $\mathbb{R}$ of real numbers, $\theta$ is the zero of $X$ as a vector space.

We apply RCD method to approximate $\mathbf{s}$, only the RC itself becomes more "constructible" due to the usage of bases and coordinates. Most of the reasonings above for metric spaces remain, with usual $\rho(\mathbf{x} ; \mathbf{y})=\|\mathbf{x}-\mathbf{y}\|$.

We keep the constraints $\$ 1-3$. As for $\$ \mathrm{M} 1$, it is provided by

\&N1. $X$ is finite-dimensional: $\operatorname{dim} X=m \in \mathbb{N}$.

- $X$ is a complete (Banach) space.

- If $A \subseteq X$ is closed and bounded $(A \subseteq B[\theta ; R])$, then $A$ is compact.

In particular, any closed ball is compact - $\mathbf{\$ M} 1$.

- If $L$ is a (linear) subspace of $X(L<X)$, then $L$ is a closed subspace.

- If $L<X$ and $\mathbf{x} \notin L$, then $\rho(\mathbf{x} ; L)=\inf _{\mathbf{u} \in L}\|\mathbf{x}-\mathbf{u}\|>0$ and $\mathbf{h} \in L$ exists such that $\|\mathbf{x}-\mathbf{h}\|=\rho(\mathbf{x} ; L)$. 
We could take any normalized basis $E=\left\{\mathbf{e}_{j}\right\}_{j=1}^{m}$ of $X$ (that is, $\left\|\mathbf{e}_{j}\right\| \equiv 1$, $E$ is linearly independent, and $\left.X=L(E)=\left\{\sum_{j=1}^{m} x_{j} \mathbf{e}_{j} \mid x_{j} \in \mathbb{R}\right\}\right)$; however, for the sake of optimization of the RC we prefer the so-called Auerbach bases.

We denote by $X^{*}$ the dual, or adjoint, space of $X$, that is, the space of all linear bounded functionals $f: X \rightarrow \mathbb{R}$. $\|\cdot\|_{*}$ is the norm of $X^{*}$.

- For any $f \in X^{*}$ and any $\mathbf{x} \in X,|f(\mathbf{x})| \leqslant\|f\|_{*} \cdot\|\mathbf{x}\|$.

Auerbach theorem (see [3] and [20], Proposition 20.12). There exist $\left\{\mathbf{e}_{j}\right\}_{j=1}^{m} \subset X$ and $\left\{f_{j}\right\}_{j=1}^{m} \subset X^{*}$ such that $\left\|\mathbf{e}_{j}\right\|=\left\|f_{j}\right\|_{*}=1$ for $j=\overline{1, m}$ (normality) and $f_{i}\left(\mathbf{e}_{j}\right)=\delta_{i j}$ for $i, j=\overline{1, m}$ (biorthogonality).

Let $\mathbb{E}=\left\{\mathbf{e}_{j}\right\}_{j=1}^{m}$ from Auerbach theorem. For any $\mathbf{e}_{j}$ and any $\mathbf{u}=$ $\sum_{i \neq j} u_{i} \mathbf{e}_{i} \in L_{-j}=L\left(\mathbb{E} \backslash\left\{\mathbf{e}_{j}\right\}\right)$ we have

$1=\left|\delta_{j j}-0\right|=\left|f_{j}\left(\mathbf{e}_{j}\right)-\sum_{i \neq j} u_{i} f_{j}\left(\mathbf{e}_{i}\right)\right|=\left|f_{j}\left(\mathbf{e}_{j}-\mathbf{u}\right)\right| \leqslant\left\|f_{j}\right\|_{*} \cdot\left\|\mathbf{e}_{j}-\mathbf{u}\right\|=\left\|\mathbf{e}_{j}-\mathbf{u}\right\|$.

Thus $\rho\left(\mathbf{e}_{j} ; L_{-j}\right) \geqslant 1$. On the other hand, $\theta \in L_{-j}$ and $\left\|\mathbf{e}_{j}-\theta\right\|=1$, so $\rho\left(\mathbf{e}_{j} ; L_{-j}\right)=\left\|\mathbf{e}_{j}\right\|=1, \quad j=\overline{1, m}$,

- in addition to being the normalized basis of $X(\mathbb{E}$ is linearly independent and $|\mathbb{E}|=\operatorname{dim} X)$ this Auerbach basis $\mathbb{E}$ has the "orthogonality" property.

See also [28, Section 11.1.3, pp. 517-519]. If we have some non-Auerbach basis $E$ of $X$ and want to "construct", or approximate, the Auerbach one $\mathbb{E}$ (i.e., calculate the coordinates of $\mathbf{e}_{j} \in \mathbb{E}$ in $E$ ) using the referenced "canonical" Ruston's proof, which involves the maximization of the determinant, then we can search for that maximum in the $m^{2}$-dimensional space of the coordinates of the $m$-tuples of the points on $\{\mathbf{x} \in X:\|\mathbf{x}\|=1\}-$ a complicated task as $m$ increases; on the other hand, we perform it only once for given $(X ;\|\cdot\|)$.

Refining cover. We describe (or just recall) the cover of $B=B[\theta ; 1]$ by the "lattice" of the closed balls of radius $\frac{1}{2}$ (compare with [13, Sections 2.2 , $6.3])$.

Let $\mathbf{x} \in B$ and $\mathbf{x}=\sum_{j=1}^{m} x_{j} \mathbf{e}_{j}$. For $x_{j} \neq 0$

$$
1 \geqslant\|\mathbf{x}\|=\left|x_{j}\right| \cdot\left\|\mathbf{e}_{j}+\sum_{i \neq j} \frac{x_{i}}{x_{j}} \mathbf{e}_{i}\right\| .
$$

Since $\left(-\sum_{i \neq j} \frac{x_{i}}{x_{j}} \mathbf{e}_{i}\right) \in L_{-j}=L\left(\left\{\mathbf{e}_{1} ; \ldots ; \mathbf{e}_{j-1} ; \mathbf{e}_{j+1} ; \ldots ; \mathbf{e}_{m}\right\}\right)$, we obtain

$$
1 \geqslant\left|x_{j}\right| \cdot \rho\left(\mathbf{e}_{j} ; L_{-j}\right) \text {. }
$$

By construction of $\mathbb{E}$, we have $(5): \rho\left(\mathbf{e}_{j} ; L_{-j}\right)=1$, therefore $\left|x_{j}\right| \leqslant 1$. Let $c_{i}=-1+\frac{i}{m}, i=\overline{0,2 m}$ : break $[-1 ; 1]$ into $\left[c_{i} ; c_{i+1}\right]$ of length $\frac{1}{m}$. 
Consider the set of the balls

$$
\mathcal{B}=\left\{B\left[\mathbf{c} ; \frac{1}{2}\right] \mid \mathbf{c}=\sum_{j=1}^{m} c_{i_{j}} \mathbf{e}_{j}, i_{j}=\overline{0,2 m}, j=\overline{1, m}\right\} .
$$

There are $(2 m+1)^{m}$ of them, and we remove from $\mathcal{B}$ the balls $B^{\prime}=B\left[\mathbf{c} ; \frac{1}{2}\right]$ such that $\|\mathbf{c}\|>\frac{3}{2}$, because $B^{\prime} \cap B=\varnothing$ (if $\mathbf{x} \in B^{\prime} \cap B$, then $\|\mathbf{c}\| \leqslant$ $\left.\|\mathbf{c}-\mathbf{x}\|+\|\mathbf{x}\| \leqslant \frac{1}{2}+1\right)$.

We claim that, for any $\mathbf{x} \in B$, $\mathbf{c}$ exists such that $\mathbf{x} \in B\left[\mathbf{c} ; \frac{1}{2}\right] \in \mathcal{B}$. To obtain such $\mathbf{c}$, we take $c_{i_{j}}$ that is closest to $x_{j}\left(i_{j}=\operatorname{rnd}\left(m\left(1+x_{j}\right)\right)\right.$, where $\operatorname{rnd}(x)=\lfloor x\rfloor+\lfloor 2\{x\}\rfloor=\lfloor 2 x\rfloor-\lfloor x\rfloor)$. Then $\left|x_{j}-c_{i_{j}}\right| \leqslant \frac{1}{2 m}$ and

$$
\|\mathbf{x}-\mathbf{c}\| \leqslant \sum_{j=1}^{m}\left|x_{j}-c_{i_{j}}\right| \cdot\left\|\mathbf{e}_{j}\right\| \leqslant \sum_{j=1}^{m} \frac{1}{2 m}=\frac{1}{2} \text {. }
$$

Thus $\mathcal{B}$ is the cover of $B$. Scaled and translated $\breve{\mathcal{B}}=\mathbf{z}+r \mathcal{B}=\{B[\mathbf{z}+$ $\left.\left.\mathbf{c} ; \frac{r}{2}\right]: B\left[\mathbf{c} ; \frac{1}{2}\right] \in \mathcal{B}\right\}$ is the sought cover of $B[\mathbf{z} ; r]$, at that for any $B\left[\mathbf{z}^{\prime} ; \frac{r}{2}\right] \in \breve{\mathcal{B}}$ : $\left\|\mathbf{z}^{\prime}-\mathbf{z}\right\| \leqslant \frac{3}{2} r$ (compare with Proposition 1.6).

\section{Non-Euclidean SR examples ${ }^{1}$}

1. In a city on a plain whose streets form a rectangular grid, water-main breach occurs at some address and the water begins to spread along the streets, flooding the city. We know when the water reached some points (e.g., few CCTVs are scattered at the crossroads) and want to locate the breach. In this example $X$ is the set of addresses and $\rho(\mathbf{a} ; \mathbf{b}) \approx\left|x_{a}-x_{b}\right|+\left|y_{a}-y_{b}\right|$, which is the so-called "Manhattan distance". See [8], [12].

2. In a social network (graph), some users (vertices) are connected by "friend"-like links (edges). One user shares a "sensation" with "friends", who in turn spread it among theirs, etc. Assuming that we know when certain users learnt the "sensation", we seek who caused a big stir. $X$ consists of all users and $\rho(\mathbf{a} ; \mathbf{b})$ is the shortest time needed by an information to travel between $\mathbf{a}$ and $\mathbf{b}$ (multiplied by the speed of spreading). See [24], [36].

3. In a desert hilly place with a scarce but connected vegetation, where the wind is absent or faint, the fire starts due to the arson. Few inhabitants have reported the time when the fire reached their dwellings. Knowing these points in time and the coordinates of the dwellings, we want to find where and when the arson happened. $X$ is the vegetated area, and $\rho(\mathbf{a} ; \mathbf{b})$ is the length of the shortest path through this area from $\mathbf{a}$ to $\mathbf{b}$. See [26], [27].

4. In a number system $X$, e.g., $\mathbb{N}, \operatorname{GF}\left(p^{n}\right), \mathbb{Q}_{p}, \mathbb{Z}[\zeta]$ (see [19, Chapter 7], $[29$, Sections II.1, I.6]), when the entity $\mathfrak{E}$ with given computational power establishes a property $\mathcal{P}$, say, (non)primality, of the number a, it takes certain amount of time for $\mathfrak{E}$ to establish $\mathcal{P}$ for another number $\mathbf{b}$, and vice

\footnotetext{
${ }^{1} 1$ st and 2 nd examples were provided by the referee who suggested this section.
} 
versa. We know that $\mathfrak{E}$ began from a single number $\mathbf{s}$ and the moments when it established $\mathcal{P}$ for other numbers, which are known as well. What is s? See [21], [39].

\section{Remarks}

1. Defects. Other defect functions

$$
\begin{gathered}
D_{1}(\mathbf{x})=\frac{2}{n^{2}} \sum_{1 \leqslant i<j \leqslant n}\left|\tau_{i}(\mathbf{x})-\tau_{j}(\mathbf{x})\right|, \quad D_{2}(\mathbf{x})=\frac{1}{n} \sum_{i=1}^{n}\left[\tau_{i}(\mathbf{x})-\frac{1}{n} \sum_{j=1}^{n} \tau_{j}(\mathbf{x})\right]^{2}, \\
D_{\infty}(\mathbf{x})=I(\mathbf{x})=\max _{i} \tau_{i}(\mathbf{x})-\min _{i} \tau_{i}(\mathbf{x})=\max _{1 \leqslant i<j \leqslant n}\left|\tau_{i}(\mathbf{x})-\tau_{j}(\mathbf{x})\right|, \ldots
\end{gathered}
$$

have properties similar to those of $D(\cdot)$. For instance,

$$
\left|D_{2}(\mathbf{x})-D_{2}(\mathbf{y})\right| \leqslant 8 \max _{i, j} \rho\left(\mathbf{r}_{i} ; \mathbf{r}_{j}\right) \cdot \rho(\mathbf{x} ; \mathbf{y}) \text {. }
$$

2. Issues with gradient method (GM) of searching for the minima of the defect function $f_{D}(\mathbf{x})$, which starts at the initial point $\mathbf{x}_{0}$ and "moves" in the direction of the steepest descent (another name of this method) of $f_{D}(\mathbf{x})$ (see [6, Section 16.3], [30, Chapter 25]). Obviously, $\mathbf{s}$ is a local (and global) minimum, locmin, of $f_{D}(\cdot)$. However, in general, there can be more than one locmin, even without noise (compare with [9, Section 5.2], [2, Section $9.4])$.

Example 2.1. Consider the defect $D_{2}(\cdot)$ - the variance of the random variable with equiprobable values $\tau_{i}(\mathbf{x})$. Let $(X ; \rho)=\mathbb{R}_{2}^{2}, \mathbf{s}=(0 ; 0)$, and 5 sensors: $\mathbf{r}_{1}=(8 ; 6), \mathbf{r}_{2}=(5 ; 5), \mathbf{r}_{3}=(-2 ; 6), \mathbf{r}_{4}=(-6 ; 4), \mathbf{r}_{5}=(-10 ; 2)$.

$\triangleleft$ Numerical experiments show that there is the locmin of $D_{2}(\mathbf{x})$ at $\mathbf{b} \approx$ $(-3.6901 ; 21.5627)$, at that $D_{2}(\mathbf{b}) \approx 0.69044$. Therefore we cannot start GM at arbitrary initial point to search for the solution. We remark that [15, Proposition 4] implies uniqueness of the solution s, since $\mathbf{r}_{5}=2 \mathbf{r}_{4}-\mathbf{r}_{3}$. $\triangleright$

Similar configurations exist for higher dimensionalities.

Moreover, GM that starts at the sensor nearest to s can converge to the $\operatorname{locmin} \mathbf{b} \neq \mathbf{s}$.

Example 2.2. Let $(X ; \rho)=\mathbb{R}_{2}^{2}, \mathbf{s}=(0 ; 0)$, and 5 sensors: $\mathbf{r}_{1}(1.885 ; 0.014)$, $\mathbf{r}_{2}(2.523 ;-0.76), \mathbf{r}_{3}(2.552 ;-0.756), \mathbf{r}_{4}(2.94 ;-0.78), \mathbf{r}_{5}(2.081 ; 0.986)$

$\triangleleft \mathrm{GM}$ with the initial point $\mathbf{r}_{1}$, which is nearest to $\mathbf{s}$, converges to the locmin of $D_{2}(\mathbf{x})$ at $\mathbf{b} \approx(2.039 ; 0.253), D_{2}(\mathbf{b}) \approx 0.00318$. $\triangleright$

Again, similar behaviour can occur in $\mathbb{R}_{2}^{m}$ for $m>2$.

3. Towards Noise. When, instead of exact $t_{i}$, we know only "shifted" $\widehat{t_{i}}=t_{i}+\xi_{i}$ and $\widehat{\tau}_{i}(\mathbf{x})=\widehat{t_{i}}-\rho\left(\mathbf{x} ; \mathbf{r}_{i}\right)$ (noises $\xi_{i}$ are supposed to be random variables with certain properties $), \widehat{D}=\frac{1}{n^{2}} \sum_{i}\left|\sum_{j}\left(\widehat{\tau}_{i}-\widehat{\tau}_{j}\right)\right|$ is "distorted"; 
we lose Proposition 1.1, Proposition 1.4, and what is built on top of them. Maybe $\widehat{D}$ has a continuum of zeros, maybe none.

If we opt to keep using the root finding approach rather than the optimization one, then the following "trick" may be applied, assuming $\left|\xi_{i}\right| \leqslant \gamma$ for small enough $\gamma$ : consider $\widetilde{D}=|\widehat{D}-2 \gamma|$.

It is easy to see that $|\widehat{D}-D| \leqslant 2 \gamma$, thus $\widehat{D}(\mathbf{s})-2 \gamma \leqslant 0$, while at some distant x presumably $\widehat{D}(\mathbf{x})-2 \gamma>0 ; \widetilde{D}$ has zeros. Like $\widehat{D}, \widetilde{D}$ is a "distortion" of $D$, but due to $\gamma \approx 0 \widetilde{D}$ should be small enough for the (continuum of) zeros of $\widetilde{D}$ to be "not too far" from s. These zeros form the closed (topologically and geometrically) "surface(s)" $Z=\{\mathbf{x} \in X \mid \widetilde{D}(\mathbf{x})=0\}$ around or near s; in a sense, the distortion "inflates" single zero, turning it into surface(s).

Proposition 1.3 remains valid for $\widetilde{D}$, and instead of Proposition 1.4 we shall have its analogue with $\rho(\mathbf{x} ; \mathbf{s})$ replaced by $\rho(\mathbf{x} ; Z)$. We use the test " $2 r_{k}<\widetilde{D}(\mathbf{c})$ ", and the refining cover $\left\{\mathcal{C}_{k}\right\}_{k=0}^{\infty}$ consisting of $B\left[\mathbf{c} ; r_{k}\right]$ that do not satisfy this inequality "converges" to $Z$, which stays mostly within the union of the balls from $\mathcal{C}_{k}$. At some iteration we halt and take some point "between" the centers of these balls (their mean in normed space, for instance)... and rely on this point being close enough to $\mathbf{s}$.

The locmins cause one of evident drawbacks of this trick: false, or "ghost", solutions can appear near such minima, relatively far from the true source $\mathbf{s}$. At least they should not appear, and $\mathcal{C}_{k}$ should not break into the disjoint groups of the balls as $k \rightarrow \infty$, if $2 \gamma<\mu$, where $\mu$ is the minimal value of $\widehat{D}(\mathbf{b})$ at the locmins $\mathbf{b}$ that are not the "descendants" of $\mathbf{s}$.

Meta-refinement: we run, in parallel, several instances of $|\widehat{D}-\lambda|$-trick with different $\lambda$ (e.g., $\lambda_{i j}= \pm \frac{i}{2^{j-1}} \gamma$ ), compare how the respective covers behave, and spawn new instances if needed.

\section{Conclusions}

We have considered the SRP in proper metric spaces and, under some assumptions, described the algorithm to approximate its solution to preselected precision. The method can be implemented on a general purpose computer (see Appendix), although we do not claim that it is optimal in all such spaces. In the finite-dimensional normed spaces, the cover that the algorithm uses is built in a more constructive way. The algorithm can be adjusted for noised measurements.

Future work: obtain estimates of the time in which the algorithm executes based on properties of the space, e.g., the doubling constant; look into optimal arrangement of sensors; estimate memory requirements; study robustness more thoroughly; consider non-proper spaces. 


\section{Appendix}

The implementation of RCD algorithm with the defect $D_{\infty}(\cdot)$ for $\mathbb{R}_{p}^{m}$, where $m \in \mathbb{N}, 1 \leqslant p<\infty$, in Julia language (see julialang.org).

space.jl

module Space

\#\# Primary constants

const $\mathrm{DIM}=2 \# \mathrm{~m}$

const POW $=5.6789 \# \mathrm{p}$

\#\# Derived constants

\# We cover ball or radius 1 by balls of radius $1 / 2$.

\# Their centers are at nodes of (hyper)cubic lattice.

\# The number $\mathrm{N}$ of small cubes along each axis must be big enough

\# so that the longest diagonal of a small cube is not longer than 1 .

const COVER_AXIS_STEP_NUM $=\operatorname{Int}\left(\operatorname{ceil}\left(2.0 /\left((1.0 / \mathrm{DIM})^{-}(1 / \mathrm{POW})\right)\right)\right)$

const COVER_AXIS_STEP_LEN $=2.0 /$ COVER_AXIS_STEP_NUM

struct Point

$\mathrm{x}:$ : Array $\{\mathrm{Float64,1 \}}$

end

struct Coverand

center: : Point

radius : :Float64

end

struct CoverandIndex

i: : Array $\{$ Int, 1$\}$

end

export Point, Coverand

distance $\left.(\mathrm{p} 1, \mathrm{p} 2)=\left(\operatorname{sum}((\operatorname{abs} .(\mathrm{p} 1 . \mathrm{x}-\mathrm{p} 2 . \mathrm{x})))^{\wedge} \mathrm{POW}\right)\right)^{\wedge}(1.0 / \mathrm{POW})$

newZeroPoint ()$=\operatorname{Point}($ zeros $($ Float64, DIM $))$

newRandomPoint $(\mathrm{rad}=1.0)=$ Point $(\operatorname{rad} *(1.0 .-2.0 * \operatorname{rand}($ Float64, DIM $)))$

newCoverandIndex ()$=\operatorname{CoverandIndex}(\operatorname{zeros}(\operatorname{Int}, \mathrm{DIM}))$

\# Increment d-digit number in base COVER_AXIS_STEP_NUM+1

\# (i-th digit is the index of cubic lattice node along $i$-th axis)

function nextCoverandIndex (index)

next $=$ deepcopy (index)

carry, $\operatorname{dim}=1,1$

while $($ carry $==1)$ \&\& $(\operatorname{dim}<=$ DIM $)$

next.i[dim] $+=$ carry

if (next.i[dim] > COVER_AXIS_STEP_NUM)

next.i[dim], carry $=0,1$

else 


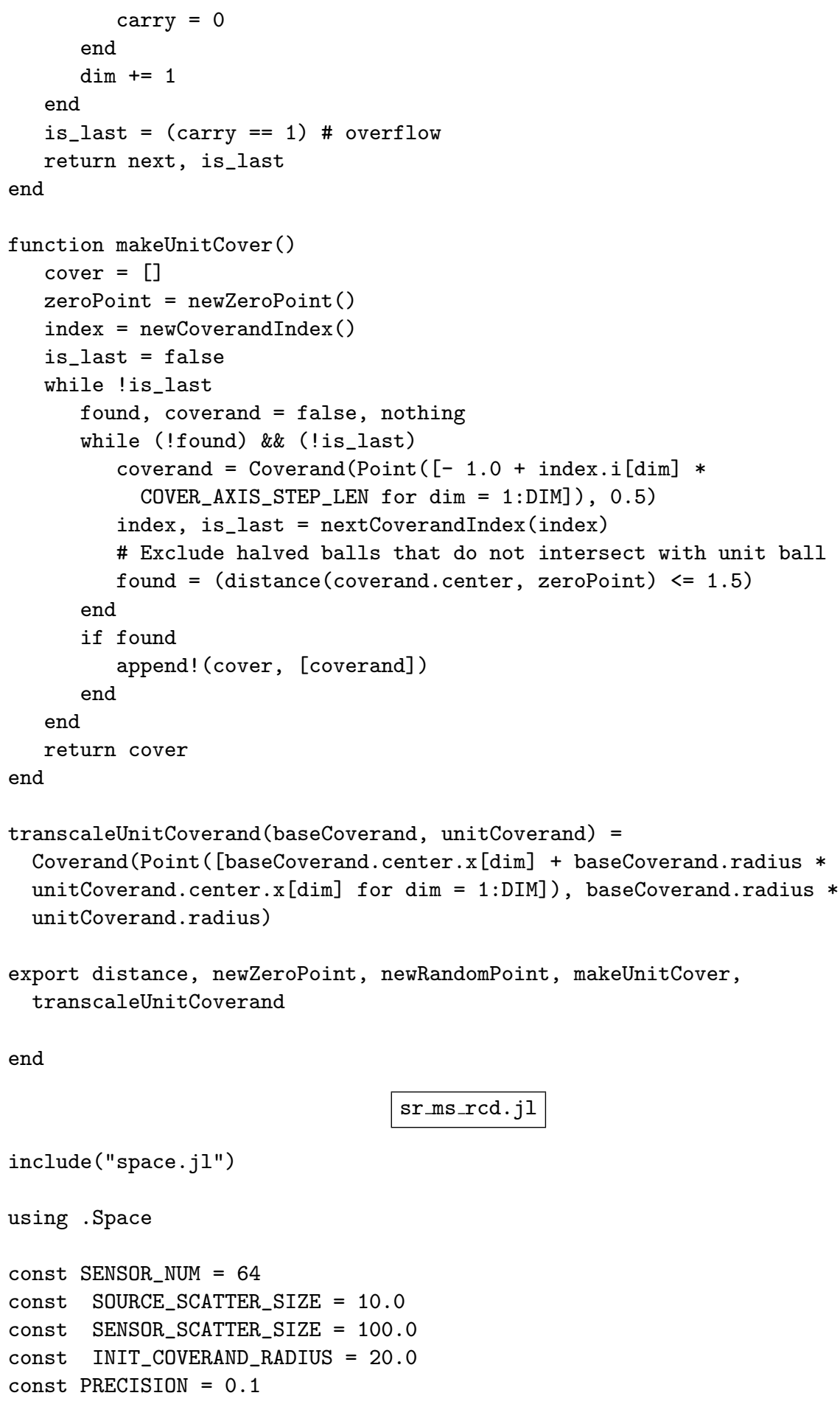




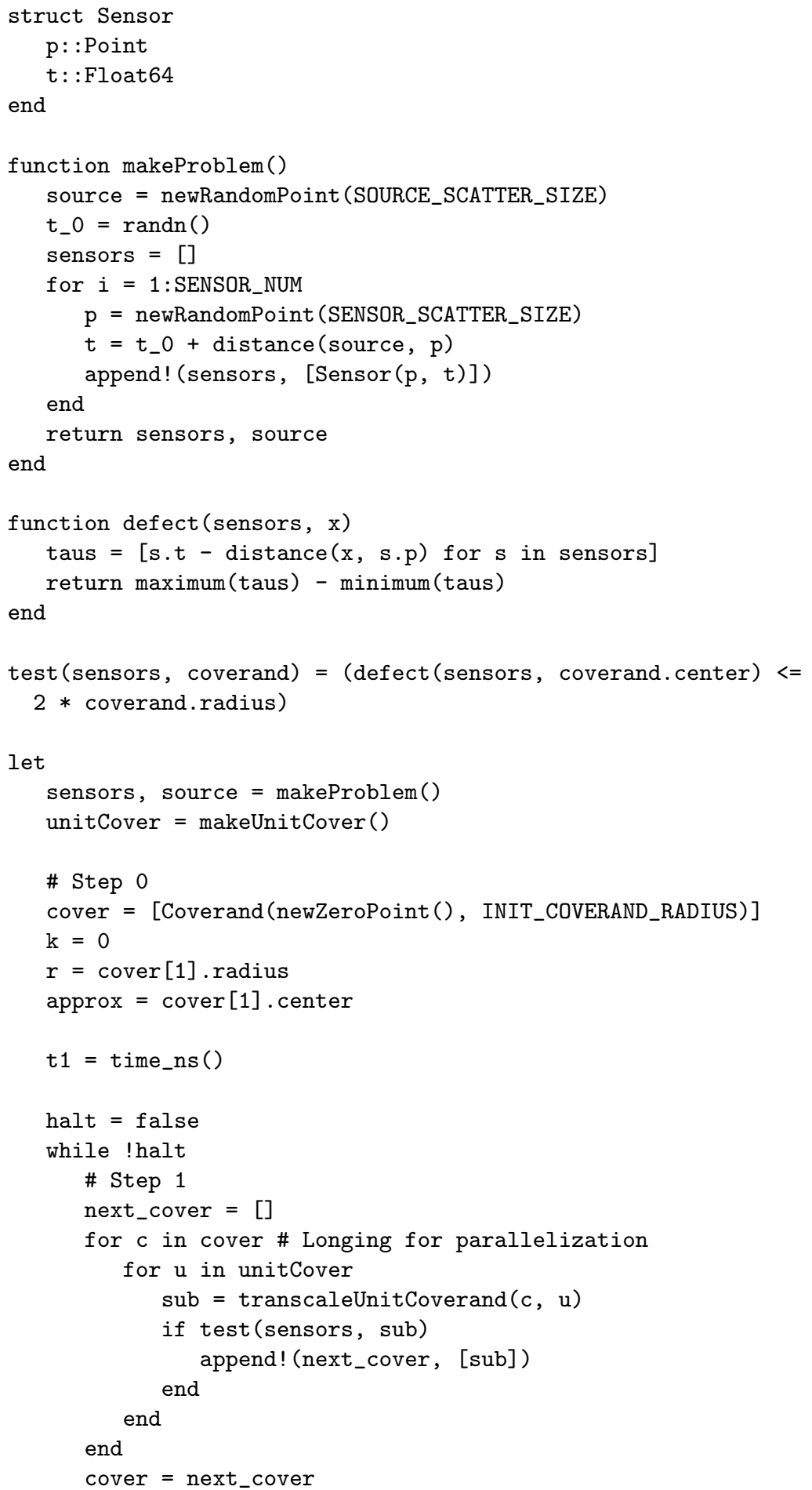




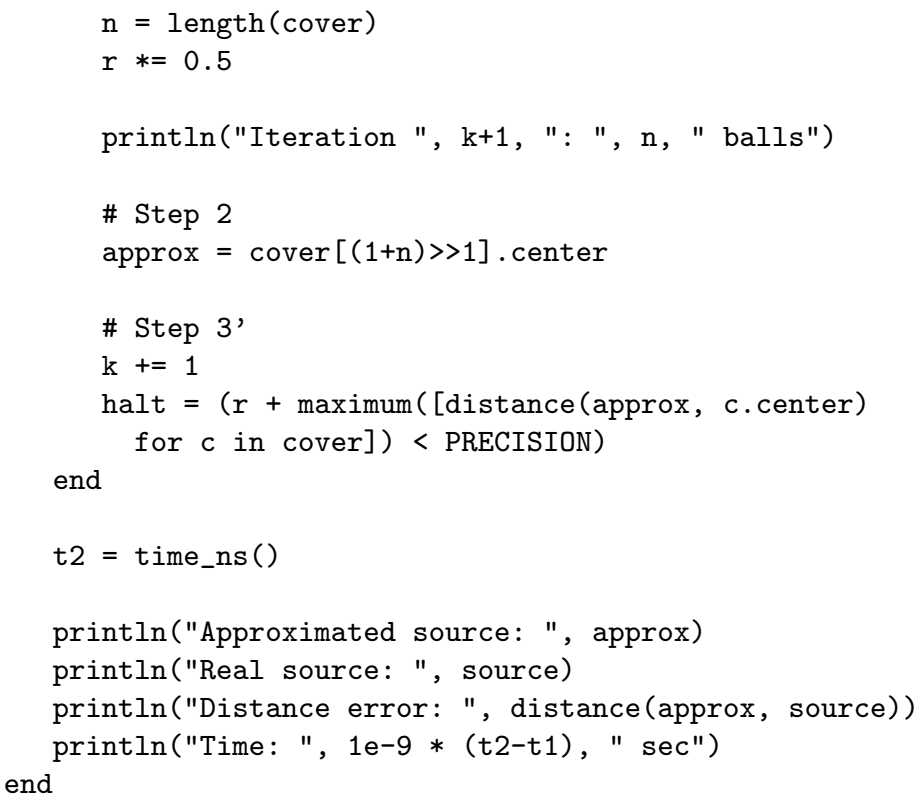

Typical execution result for $m=2, p=5.6789,64$ sensors, and $\delta=0.1$ is shown below. Note that $(+)$ the distance error is smaller than the precision $\delta$ and $(-)$ the number of balls in a cover does not decrease.

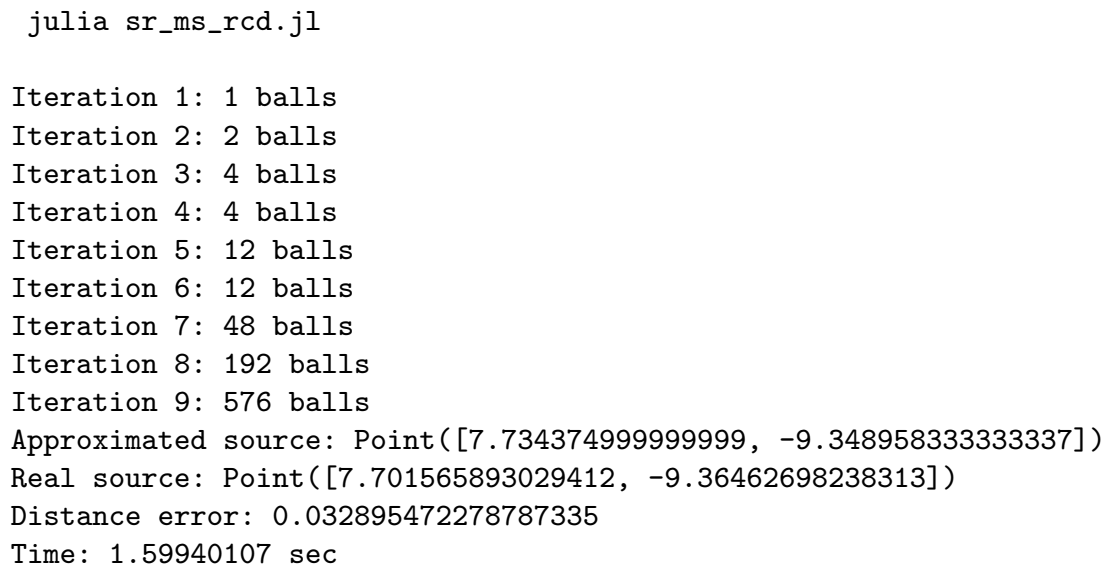

\section{Acknowledgements}

We sincerely thank anonymous referees, whose reports have ameliorated the paper substantially; in particular, they helped to improve the presentation, to make it more concluded, and to clarify the notions regarding the applicability. 


\section{References}

[1] X. Alameda-Pineda and R. Horaud, A geometric approach to sound source localization from time-delay estimates, IEEE Trans. Audio Speech Lang. Process. 22 (2014), 10821095.

[2] Audio Signal Processing for Next-Generation Multimedia Communication Systems (Edited by Y. Huang and J. Benesty), Kluwer Academic Publishers, 2004.

[3] H. Auerbach, On the area of convex curves with conjugate diameters, Ph.D. thesis, University of Lwów, 1930. (Polish)

[4] S. Bancroft, An algebraic solution of the GPS equations, IEEE Trans. Aerosp. Electr. Syst. 21(1) (1985), 56-59.

[5] P. Bestagini, M. Compagnoni, F. Antonacci, A. Sarti, and S. Tubaro, TDOA-based acoustic source localization in the space-range reference frame, Multidim. Syst. Sign. Process. 25(2) (2014), 337-359.

[6] C. L. Byrne, Applied Iterative Methods, A K Peters, Ltd., Wellesley, MA, 2008.

[7] J. C. Chen, R. E. Hudson, and K. Yao, Maximum-likelihood source localization and unknown sensor location estimation for wideband signals in the near-field, IEEE Trans. Sign. Process. 50(8) (2002), 1843-1854.

[8] W. Chiu and B. Chen, Mobile positioning problem in Manhattan-like urban areas: uniqueness of solution, optimal deployment of BSs, and fuzzy implementation, IEEE Trans. Sign. Process. 57(12) (2009), 4918-4929.

[9] M. Compagnoni, A. Canclini, P. Bestagini, F. Antonacci, A. Sarti, and S. Tubaro, Source localization and denoising: a perspective from the TDOA space, Multidim. Syst. Sign. Process. 28(4) (2017), 1283-1308.

[10] M. M. Deza and E. Deza, Encyclopedia of Distances, Springer-Verlag, Berlin, 2009.

[11] I. Dumer, Covering spheres with spheres, Discrete Comput. Geom. 38(4) (2007), 665-679.

[12] K. El Kadi Abderrezzak, A. Paquier, and E. Mignot, Modelling flash flood propagation in urban areas using a two-dimensional numerical model, Nat. Hazards 50 (2009), 433-460.

[13] J. M. Fowkes, N. I. M. Gould, and C. L. Farmer, A branch and bound algorithm for the global optimization of Hessian Lipschitz continuous functions, J. Glob. Optim. 56(4) (2013), 1791-1815.

[14] M. D. Gillette and H. F. Silverman, A Linear Closed-Form Algorithm for Source Localization From Time-Differences of Arrival, IEEE Sign. Process. Lett. 15 (2008), $1-4$.

[15] S. V. Goncharov, On sound ranging in Hilbert space, Note Mat. 38(1) (2018), 47-65.

[16] Handbook of Discrete and Computational Geometry (Edited by J. E. Goodman, J. O'Rourke, and C. D. Tóth), CRC Press, Boca Raton, FL, 2018.

[17] J. Heinonen, Lectures on Analysis on Metric Spaces, Springer-Verlag, New York, 2001.

[18] J. E. Hopcroft, R. Motwani, and J. D. Ullman, Introduction to Automata Theory, Languages, and Computation, Addison Wesley, 2006.

[19] K. Ireland and M. Rosen, A Classical Introduction to Modern Number Theory, Springer-Verlag, New York, 1990.

[20] G. J. O. Jameson, Topology and Normed Spaces, Chapman and Hall, London, 1974.

[21] P. C. Kocher, Timing attacks on implementations of Diffie-Hellman, RSA, DSS, and other systems, in: Advances in Cryptology-CRYPTO'96, Lecture Notes in Computer Science 1109 (1996), pp. 104-113.

[22] A. N. Kolmogorov and S. V. Fomin, Introductory Real Analysis, Dover Publications, Inc., New York, 1975. 
[23] H. Lebesgue, Letters of Henri Lebesgue to Emile Borel, Cahiers Sém. d'Hist. Math. 12 (1991), 1-506. (French)

[24] A. Louni and K. P. Subbalakshmi, Who spread that rumor: finding the source of information in large online social networks with probabilistically varying internode relationship strengths, IEEE Trans. Comput. Soc. Syst. 5(2) (2018), 335-343.

[25] G. Mao and B. Fidan, Localization Algorithms and Strategies for Wireless Sensor Networks, Information Science Reference, Hershey, 2009.

[26] A. B. Massada, A. D. Syphard, T. J. Hawbaker, S. I. Stewart, and V. C. Radeloff, Effects of ignition location models on the burn patterns of simulated wildfires, Env. Model. \& Soft. 26(5) (2011), 583-592.

[27] S. Monedero, J. Ramirez, D. Molina-Terrén, and A. Cardil, Simulating wildfires backwards in time from the final fire perimeter in point-functional fire models, Env. Model. \& Soft. 92 (2017), 163-168.

[28] V. Montesinos, P. Zizler, and V. Zizler, An Introduction to Modern Analysis. Springer, Cham, 2015.

[29] J. Neukirch, Algebraic Number Theory, Springer-Verlag, Berlin, 1999.

[30] A. M. Ostrowski, Solutions of Equations in Euclidean and Banach Spaces, Academic Press, New York-London, 1973.

[31] A. Papadopoulos, Metric Spaces, Convexity and Non-Positive Curvature, European Mathematical Society, Zürich, 2014.

[32] P. Petersen, Riemannian Geometry, Springer, New York, 2006.

[33] M. Pollefeys and D. Nister, Direct computation of sound and microphone locations from time-difference-of-arrival data, in: Proceedings of International Conference on Acoustics, Speech, and Signal Processing, 2008.

[34] W. Schriever, Sound ranging in a medium having an unknown constant phase velocity, Geophysics 17(4) (1952), 915-923.

[35] M. Ó Searcóid, Metric Spaces, Springer-Verlag London, Ltd., London, 2007.

[36] D. Shah and T. Zaman, Detecting sources of computer viruses in networks: theory and experiment, SIGMETRICS Perform. Eval. Rev. 38(1) (2010), 203-214.

[37] The War of Guns and Mathematics (Edited by D. Aubin and C. Goldstein), History of Mathematics 42, American Mathematical Society, Providence, RI, 2014.

[38] W. A. Watkins and W. E. Schevill, Four-hydrophone array for acoustic threedimensional location, Tech. Report, Woods Hole Ocean. Inst., 1971.

[39] M. Weiß, B. Heinz, and F. Stumpf, A cache timing attack on AES in virtualization environments, in: Financial Cryptography and Data Security 2012, Lecture Notes in Computer Science $\mathbf{7 3 9 7}$ (2012), pp. 314-328.

[40] R. Williamson and L. Janos, Constructing metrics with the Heine-Borel property. Proc. Amer. Math. Soc. 100(3) (1987), 567-573.

Faculty of Mechanics and Mathematics, Oles Honchar Dnipro National UniVersity, 72 Gagarin Avenue, 49010 Dnipro, Ukraine

E-mail address: goncharov@mmf.dnu.edu.ua 